\title{
Surface Oxide Nanopillars Formed by Atmospheric Plasma
}

Donovan N. Leonard ${ }^{1 *}$, Michael Brady ${ }^{2}$, Harry Meyer $^{1}$, and Peter Yancey $^{3}$

1. Center for Nanophase Materials Sciences, Oak Ridge National Laboratory, Oak Ridge, TN USA.

2. Materials Science \& Technology Division, Oak Ridge National Laboratory, Oak Ridge, TN USA.

3. Atmospheric Plasma Solutions, Inc., Cary, NC USA

*Corresponding Author: leonarddn@ornl.gov

Complex interactions between a downstream atmospheric plasma (AP) discharge [1-2] and a metal surface produces visual changes (e.g., scattering and interference of light) to the surface. To better understand the morphology, composition, and chemistry of the resulting surface structures after air plasma treatment of a metal surface STEM/EELS, FESEM, AFM and XPS were used. An Inconel 718 alloy (Ni-Cr base, $\gamma$ '-strengthened superalloy) was subjected to AP processing and the surface features resulting from the plasma treatment were compared with surface features formed during a $60 \mathrm{~min}$., $800^{\circ} \mathrm{C}$ conventional resistance furnace annealing in air.

A polished Inconel 718 surface was exposed to an air atmospheric plasma operated at a power level of $2.5 \mathrm{~kW}$ for $7 \mathrm{~s}$ at a height of $15 \mathrm{~mm}$ above the metal surface (Fig. 1A), which resulted in a multi-colored region on the sample surface that measured $\sim 3 \mathrm{~mm}$ in diameter (Fig. 1B-C). The surface layer was interrogated with X-ray photoelectron spectroscopy (XPS) depth-profiling to determine the composition and chemistry within a large area of the treatment region. Low-kV field emission scanning electron microscopy (FESEM) and atomic force microscopy (AFM) were used to survey the morphology of features found in the treatment area and site-specific focused ion beam (FIB) lift-out was used to prepare cross-sectional samples for scanning transmission electron microscopy (STEM)-based imaging, energy dispersive X-ray spectroscopy (EDS), and electron energy loss spectroscopy (EELS). AFM results showed that the RMS surface roughness of the Inconel 718 increased after AP processing $(\mathrm{RMS}=9.5 \mathrm{~nm})$, which was less than that observed after furnace annealing $(\mathrm{RMS}=15.2 \mathrm{~nm})$. In-lens FESEM revealed densely packed nodular surface features measuring 15-40nm in diameter resulted from the AP processing (Fig. 2B) and faceted cube-like surface features (Fig. 2C) formed during furnace annealing. STEM bright-field images confirmed the nm-scale pillars were crystalline and that the AP treatment produced chemically distinct layers on the Inconel 718 surface (Fig. 3A). XPS and EELS (Fig. 3C) showed the layered structure consisted of a Ni- and O-rich top-most layer (45-55nm thick), an $\mathrm{Fe}$ - and O-rich layer (35-40nm thick) below this first layer, and a $\mathrm{Cr}$ - and O-rich layer (15-20nm thick) directly adjacent to the underlying Inconel 718 substrate. XPS and STEM/EDS/EELS characterization of the furnace annealed Inconel 718 showed a 20-30nm thick (Fe,Cr,O)-rich surface layer and a thicker Cr,O-rich (80-90nm) layer adjacent to the Inconel 718 substrate (Figs. 3B and 3D).

The chemically distinct oxide layers formed by the AP treatment were created within seconds using a fraction of the energy required to form a comparably thick oxide via the $60 \mathrm{~min} .800^{\circ} \mathrm{C}$ furnace anneal. The AP-treated metal surface exhibited a different morphology and elemental composition compared to the furnace annealed Inconel 718 surface. Air atmospheric plasma processing may provide a rapid and energy efficient method to grow thin oxide layers on metal substrates to enhance structural (e.g. corrosion resistance) or functional use. [3]

References: 
[1] U Kogelschatz, Plasma Chemistry and Plasma Processing 23 (2003), p. 1.

[2] L Bardos and H Barankova, Thin Solid Films 518 (2010), p. 6705.

[3] Research conducted at ORNL's Center for Nanophase Materials Sciences, which is a U.S.

Department of Energy Office of Science User Facility.
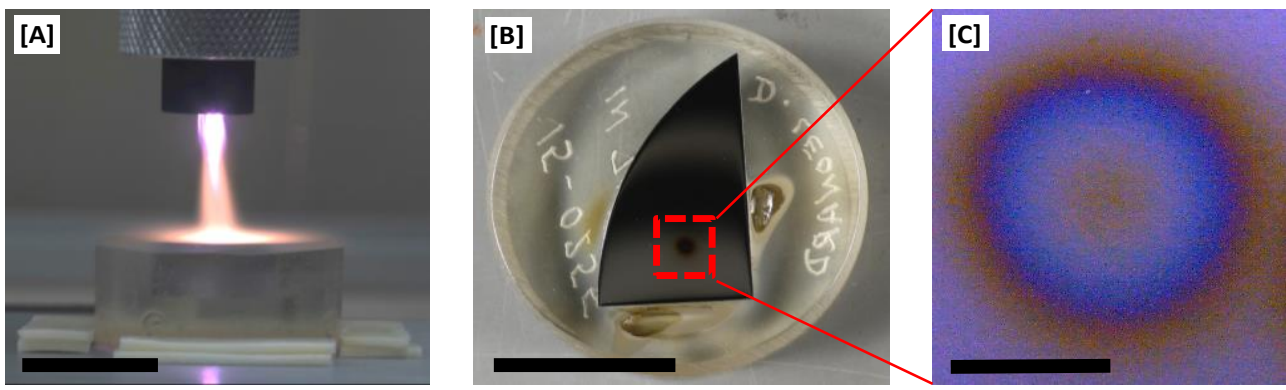

Figure 1. [A] AP plume during processing of Inconel 718. Scale bar $=20 \mathrm{~mm}$. [B] AP processed area (red box). Scale bar $=20 \mathrm{~mm}$. [C] Higher magnification of AP processed region. Scale bar $=1.5 \mathrm{~mm}$.
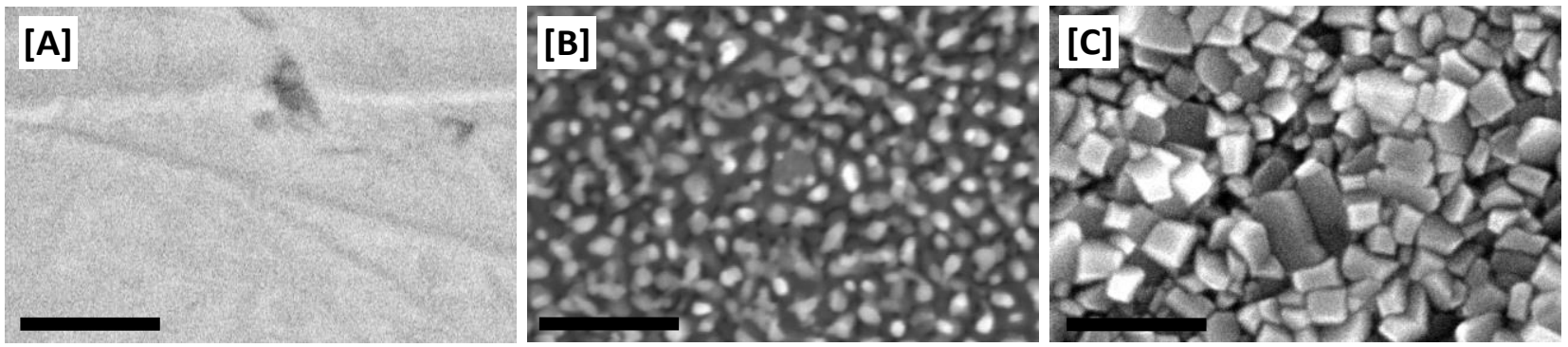

Figure 2. FE SEM images of [A] as-polished Inconel 718 surface, [B] AP processed Inconel 718 surface, $[\mathrm{C}]$ furnace annealed Inconel 718 surface (scale bars in images $=200 \mathrm{~nm}$ ).

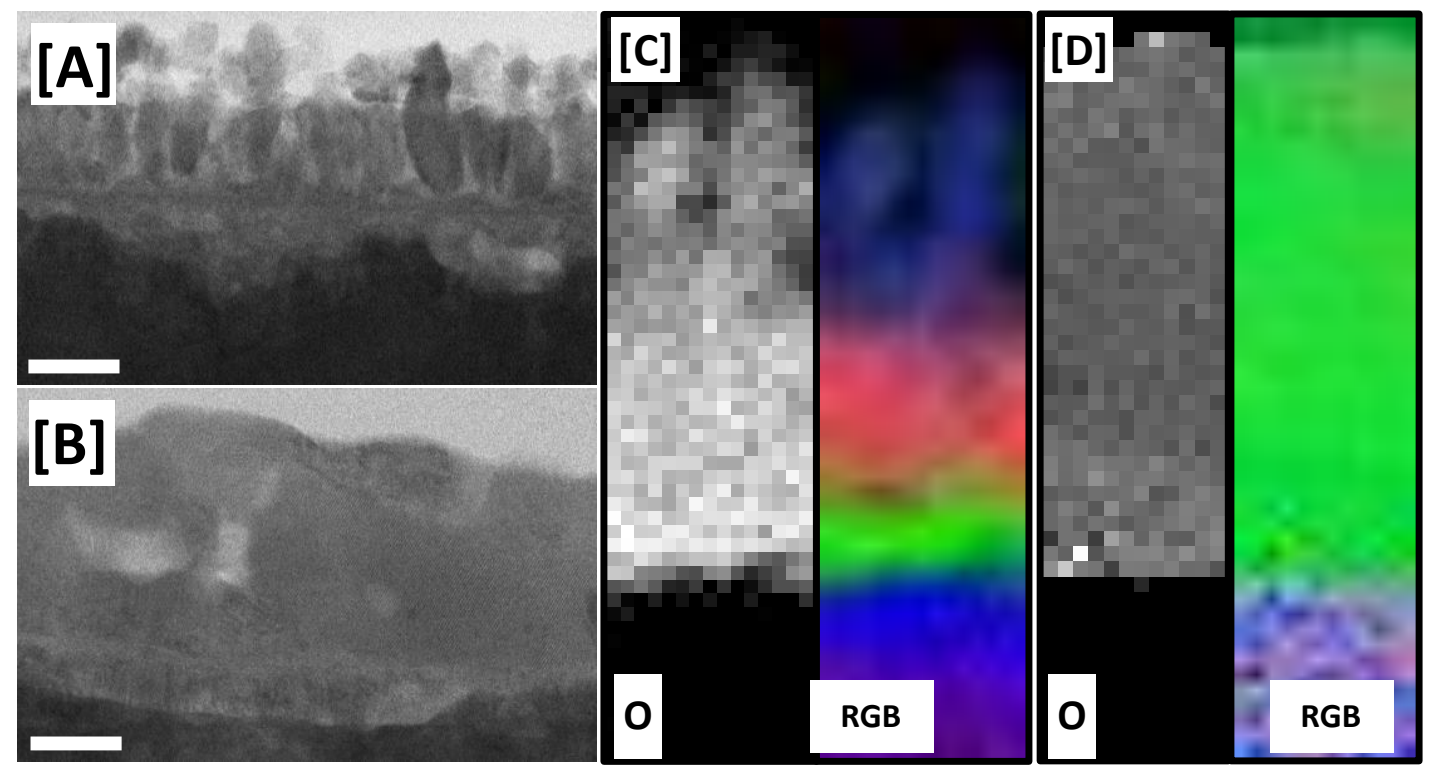

Figure 3. STEM images of cross-section of Inconel 718 surfaces. Scale bar is 50nm: [A] after AP processing and [B] after $60 \mathrm{~min} .800^{\circ} \mathrm{C}$ furnace anneal. EELS spectrum image (SI) data showing O-map and RGB map (red-Fe, green-Cr, blue-Ni): [C] from region [A] and [D] from region in [B]. 